\title{
Divertor power load studies for attached L-mode Single-Null plasmas in TCV
}

\author{
R. Maurizio ${ }^{1}$, S. Elmore ${ }^{2}$, N. Fedorczak ${ }^{3}$, A. Gallo ${ }^{3}$, H. \\ Reimerdes $^{1}$, B. Labit ${ }^{1}$, C. Theiler ${ }^{1}$, C.K. Tsui ${ }^{1}$, W.A.J. \\ Vijvers $^{4}$, the TCV Team $\ddagger$ and the MST1 Team $\S$ \\ ${ }^{1}$ École Polytechnique Fédérale de Lausanne (EPFL), Swiss Plasma Center \\ (SPC), 1015 Lausanne, Switzerland \\ 2 CCFE, Culham Science Centre, OX14 3DB Abingdon, United Kingdom \\ 3 CEA Cadarache, IRFM, F-13108 Saint-Paul-Lez-Durance, France \\ ${ }^{4}$ Dutch Institute for Fundamental Energy Research (DIFFER), 5612 AJ \\ Eindhoven, the Netherlands \\ E-mail: roberto.maurizio@epfl.ch
}

\begin{abstract}
This paper investigates the power loads at the inner and outer divertor targets of attached, Ohmic L-mode, deuterium plasmas in the TCV tokamak, in various experimental situations using an Infrared thermography system. The study comprises variations of the outer divertor leg length and target flux expansion, the plasma current and a reversal of the magnetic field direction. The direct impact of the divertor magnetic geometry on Scrape-Off Layer (SOL) transport - parameterised by the SOL power fall-off length $\lambda_{q, u}$, the divertor spreading factor $S_{u}$ and the in-out power asymmetry - is reported for constant core properties. The in-out power asymmetry increases, either with the divertor leg length, or the target flux expansion. The SOL width $\lambda_{q, u}$ scales positively with divertor leg length, with a strength that depends on the field direction and differs between the inner and outer divertor. This implies a parametric dependence of $\lambda_{q, u}$ that is not explicitly included in current multi-machine scaling laws. The divertor spreading factor at the target $S=S_{u} f_{x}$, where $f_{x}$ is the target flux expansion, appears unaffected by changes in the divertor geometry and in the plasma current, is independent of the magnetic field direction and is similar between inner and outer divertor. Possible interpretations of these observations using an ad-hoc analytical purely conductive model for the SOL, by ion drifts or by asymmetric turbulent cross-field transport in the divertor are presented. The observed values of $\lambda_{q, u}$ are related to existing L-mode and H-mode scaling laws and to similar studies in other tokamaks. Finally, potential implications of these findings for future larger fusion machines are discussed.
\end{abstract}

Keywords:

$\ddagger$ See the author list of 'S. Coda et al., Nucl. Fusion 57 (2017) 102011'

$\S$ See the author list of 'H. Meyer et al., Nucl. Fusion 57 (2017) 102014' 


\section{Introduction}

The safe operation of larger fusion experiments, such as ITER and, in particular, DEMO, will require a solution for the power exhaust issue, namely the handling of the power flowing out of the plasma through the Scrape-Off Layer (SOL) and impacting the divertor targets. The heat flux onto these targets must remain below the technologically acceptable maximum for actively cooled surfaces, which is of the order of $10 \mathrm{MW} \mathrm{m}^{-2}$ in steady state increasing to $20 \mathrm{MW} \mathrm{m}^{-2}$ for transients [1]. The peak heat flux can be mitigated either by increasing the wetted area over which the heat is spread, for example by broadening the SOL, or by increasing the power radiated in the divertor volume. The projected SOL width in ITER is based on an multi-device database [2] that predicts a small value of $\lambda_{q, \text { ITER }} \sim 1 \mathrm{~mm}$. The resulting high peak heat flux would then require a very high radiated fraction, that may exceed the capabilities of conventional divertor geometries. Increasing the confidence in scalings of the target heat flux profile and improving the understanding of the physical mechanisms that determine the SOL width remain important to the fusion community.

Recent power exhaust studies performed at the TCV tokamak may increase the confidence in present $\lambda_{q}$ scalings and provide insight into the physics of transport in the SOL. TCV's unique magnetic shaping capabilities, a highly elongated and open vacuum vessel and wide array of boundary diagnostics, make TCV ideal for investigating the impact of divertor magnetic geometry on SOL transport over a large range of plasma regimes.

This paper focuses on low-confinement plasmas (L-mode) with a Lower Single-Null (SN) divertor in the attached regime. It documents the impact of variations in the magnetic outer divertor geometry - length of the divertor leg and target flux expansion - and of the plasma current on the SOL power fall-off length $\lambda_{q}$, the divertor spreading factor $S_{u}$ and the in-out power asymmetry $P_{\text {in }} / P_{\text {out }}$.

Section 2 describes the experimental setup and strategy, the recently upgraded Infrared (IR) diagnostics and the methodology used to estimate and parameterise the target heat flux profiles from IR thermography. The experimental results are presented in section 3, organized as scans of plasma current, outer leg length, outer target flux expansion and magnetic field direction. Section 4 presents a discussion of the experimental results and introduces a simple model for heat transport in the SOL. In section 5 the results are compared with related studies on other tokamaks. The conclusions and possible implications for future larger machines are outlined in section 6 .

\section{Experimental setup and analysis}

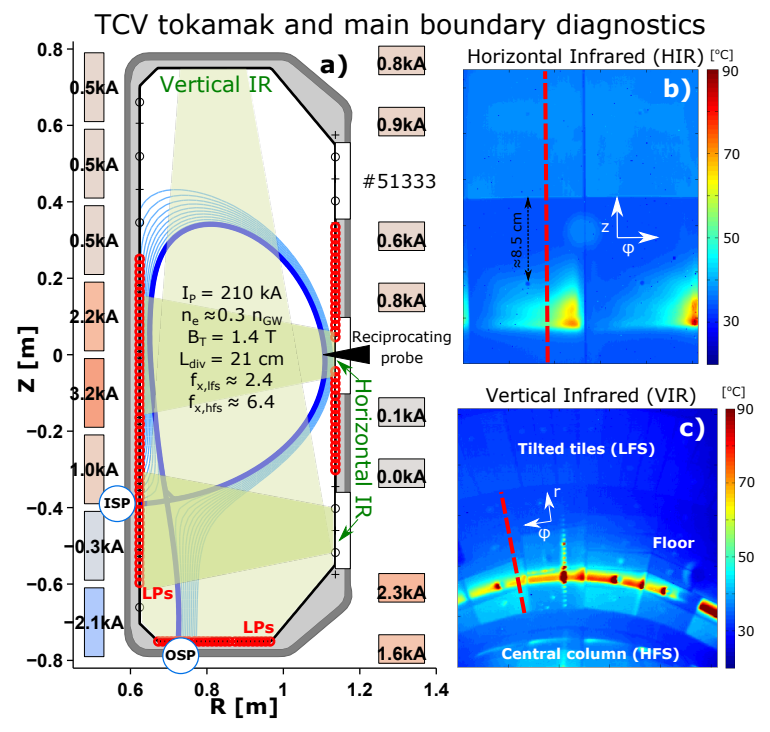

Figure 1. (a) Poloidal cross section of the TCV vessel and poloidal field coils with the flux contours of a lower Single Null. Also shown are the field-of-views of the IR cameras, the position of the mid plane reciprocating probe and the 114 wall Langmuir Probes (LPs). Typical thermal images of inner (b) and outer (c) divertor targets from IR cameras.

The Tokamak à Configuration Variable (TCV) [3] is a medium size tokamak with a major radius of $R_{0}=0.88 \mathrm{~m}$. The vessel is highly elongated and has an aspect ratio of $\approx 3$. A typical plasma pulse for the presented study has a toroidal field of $B_{0}=1.45 \mathrm{~T}$ and a plasma current up to $400 \mathrm{kA}$. TCV features 16 independently powered poloidal field coils (figure 1a), which are routinely used to generate and control a wide range of plasma shapes and divertor configurations. Over $95 \%$ of the vessel wall is covered by graphite protection tiles permitting a wide range of possible strike point locations. An Electron Cyclotron Resonance Heating (ECRH) system and a recently installed Neutral Beam Injector (NBI) are available, but were not used in this study. The 
boundary diagnostics have been recently augmented, with a Divertor Spectroscopy System (DSS [4]), a fast reciprocation probe (RCP $[5,6])$ in the outer mid-plane (OMP) and an upgraded Infrared (IR) thermography system.

The unique magnetic flexibility of TCV, together with its diagnostics array, have been used to investigate the impact of modifications in the magnetic configuration on the plasma exhaust performance. Recent studies were focused on the role of divertor geometry in the access to detachment and control of detached condition [7], featuring the Single-Null [8, 4], Snowflake divertor ( $\mathrm{SF}$ ) and alternative configurations such as the Super-X divertor [9]. In parallel, SOL transport for attached plasmas was investigated by varying several basic geometric parameters of the SN (plasma upper triangularity, outer divertor leg length, outer target flux expansion, plasma current and magnetic field direction) - and of the SF (notably, the normalised distance between the two X-points [10]).

\subsection{Characterisation of divertor heat flux profiles}

Infrared thermography system The present TCV IR thermography system consists of two fast IR cameras (figure 1). The system can simultaneously measure the divertor heat loads at each strike point for a wide range of magnetic divertor configurations.

The Vertical InfraRed system (VIR) is mounted on the top of the machine and images the vessel floor through a relay optic and covers the outer strike point (OSP) of a typical SN plasma. The camera is a Thermosensorik CMT256 MHS, sensitive in the wavelength range [1.5 to 5.1] $\mu m$ and was operated with a filter limiting the sensitivity from $[4.5$ to 5.1$] \mu m$. Typical frame rate and spatial resolution are $400 \mathrm{~Hz}$ and $2.5 \mathrm{~mm}$ in fullframe respectively. The frame rate can be increased to $15 \mathrm{kHz}$ for a sub-frame.

The Horizontal InfraRed system (HIR) is mounted on a lateral port and images a portion of the central column covering the inner strike point (ISP) of the same SN configuration. The camera is an IRCAM Equus $81 k M M W I R$, sensitive in the wavelength range [3.7 to 4.8$] \mu m$ and filtered to [4.1 to 4.8$] \mu m$. Typical frame rate and spatial resolution are $200 \mathrm{~Hz}$ and 0.8 $\mathrm{mm}$ in full frame, using a $25 \mathrm{~mm}$ focal-length lens. The HIR field-of-view can be doubled with a 12.5 mm focal-length lens, with a corresponding factor of 2 reduction in the spatial resolution. The frame rate can be increased to $30 \mathrm{kHz}$ for a sub-frame. The camera can be alternatively mounted on a lower or a mid plane port, see figure $1 \mathrm{a}$.

Both IR cameras are temperature calibrated in situ using thermocouples and heated elements embedded into dedicated carbon tiles at the vessel wall.
Target heat flux estimate Typical thermal images provided by the HIR and VIR diagnostics are shown in figure $1 \mathrm{~b}$ and $1 \mathrm{c}$. The higher temperature regions correspond to the locations of the strike points (SPs), where the magnetic separatrix intercepts the material wall and the heat flowing along the magnetic field lines impacts the targets. The discontinuities and patterns in both thermal images correspond to screws and edges of the protective carbon tiles. A vibration correction algorithm, based on phase correlation within the image, is applied to the VIR video to compensate for any relative movement between the camera and the tiles. Then, a temperature profile at a fixed toroidal location (a radial cut for the vessel floor and a vertical cut for the inner wall, see dashed lines in figures $1 \mathrm{~b}$ and $1 c)$ is selected from each video frame. The location of the profile is sufficiently distant from the screws and edges of the carbon tiles to avoid any shadowing. The temporal evolution of the surface temperature together with the thermal properties of the tiles are input to the THEODOR code [11], which solves the heat diffusion equation using the measured temperature as a boundary condition. The code calculates the heat flux impinging perpendicularly on the surface $q_{\perp}(x, t)$ (where $x$ is the radius $R$ for the VIR and the vertical position $Z$ for the HIR). Any heat diffusion in the toroidal direction is neglected by the code and a heat transmission coefficient $\alpha$ accounts for the presence of a layer of deposited material on the tile surface. The value $\alpha_{\mathrm{TCV}}=160 \mathrm{~kW} / \mathrm{m}^{2} \mathrm{~K}$ is chosen to match the calorimetric measurements based on thermocouples and to satisfy the condition of a null heat flux after the discharge terminates. The approximation of neglecting toroidal heat fluxes in the tiles holds well for the floor tiles but is less accurate for the inner column tiles, for which some toroidal temperature gradients exist (see figure 1b). These temperature gradients are due to the decrease of the curvature radius of the tile surface towards the tile edges leading to larger grazing angle of the magnetic field lines, a higher perpendicular heat flux and ultimately a higher temperature [12]. The surface curvature radius varies mainly near the edges and does not change significantly up to $3 \mathrm{~cm}$ from the central screw. Consequently the area used for the analysis is typically located $\approx 1.6 \mathrm{~cm}$ to the right of the screw.

Heat flux mapping upstream The profile of the heat flux onto the target $q_{\perp}(x)$ is the result of several mechanisms: (i) geometrical spreading due to the change in the spacing between flux surfaces, which channels heat through parallel transport from the main SOL (OMP to X-point) to the divertor targets; (ii) spreading due to cross-field transport of heat both in the main and divertor SOL (X-point to targets); (iii) 


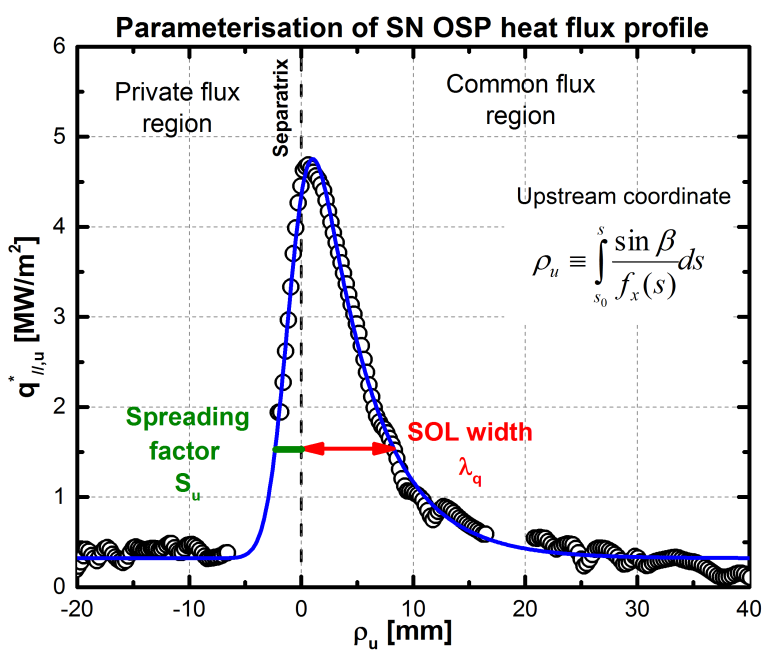

Figure 2. Typical heat flux profile given by IR for the outer target of an attached SN in TCV, projected upstream, equations 1 and 2, and interpolated with the parameterisation from [13].

radiative power losses and (iv) ion-neutral interactions in the SOL. The last two processes have a strong impact on the shape of $q_{\perp}(x)$ for partially or fully detached divertor regimes. For the study presented here, both divertors remain attached and radiative losses are small compared to the conducted power. Since a physical understanding of effect (ii) is the ultimate goal of SOL transport studies, heat flux profiles measured at the target must be corrected for geometrical spreading (i). The technique adopted in this study is to map the target heat flux profile from the SP to the outboard mid-plane in two steps.

(i) Flux surfaces in the SOL are labeled by their upstream coordinate $\rho_{u}$, defined as their outboard mid-plane distance to the separatrix. It is related to the distance along the target $s$ via

$\rho_{u}=\int_{s_{0}}^{s}\left(\sin \beta(s) / f_{x}(s)\right) d s$

where $s_{0}$ is the position of the separatrix at the target, $\beta(s)$ the poloidal angle between the field line and the target surface and $f_{x}(s)$ the target flux expansion. The flux expansion is here defined as the ratio of the distance between flux surfaces at the target $d \rho_{t}$ and upstream at the outboard mid-plane $d \rho_{u}, f_{x} \equiv d \rho_{t} / d \rho_{u}$. It is calculated as $f_{x}=\left(R_{u} B_{p, u}\right) /\left(R_{t} B_{p, t}\right)$ where $\left(R_{u} ; B_{p, u}\right)$ and $\left(R_{t} ; B_{p, t}\right)$ are radii and poloidal magnetic fields at the outer mid-plane (OMP) and at the target. The definition of $\rho_{u}$, equation 1 , is readily extended into the private flux region where it becomes negative, figure 2 , even though the location at the target is not directly magnetically connected to the OMP.

(ii) The parallel (along field lines) heat flux on the target $q_{\|, t}$ is computed by considering the field line grazing angle $\gamma$ at the target, $q_{\|, t}=q_{\perp, t} / \sin \gamma$, and is projected upstream,

$q_{\|, u}^{*}=q_{\|, t} \cdot B_{t o t, u} / B_{t o t, t}$

where the ratio $B_{t o t, u} / B_{t o t, t}$ corresponds to the change of the flux tube cross section between OMP and target. Since this is a purely geometrical projection, neither radiative losses nor cross-field transport at the target are accounted for.

Heat flux parameterisation The usual parameterisation of the heat flux profile [13] is adopted here, but applied to its upstream projection $q_{\|, u}^{*}$, see figure 2 . The fit function is the convolution of a truncated exponential with a decay length $\lambda_{q, u}$, referred to as $S O L$ power fall-off length or SOL width, with a Gaussian of width $S_{u}$, referred to as the divertor spreading factor, but mapped upstream.

The exponential is thought to characterize the heat flux profile in the SOL surrounding the confined plasma that is the sole source of power. The convolution of the truncated exponential with a Gaussian accounts for any isotropic cross-field spreading of the profile between the $\mathrm{X}$-point region and the target. In discharges with forward field (ion $\nabla B$ drift towards the active X-point), the analysis of ISP measurements is performed with an extended parameterisation of the heat flux profiles, specifically developed for TCV [14].

\subsection{Overview of the study}

This study focuses on Ohmic, L-mode deuterium discharges. The divertor is a conventional lower Single-Null, with a plasma elongation $k_{95} \approx 1.5$, an upper triangularity $\delta_{u, 95} \approx 0.24$, a lower triangularity $\delta_{l, 95} \approx 0.33$. The Greenwald fraction $f_{\mathrm{GW}}$ varies between 0.22 and 0.28 . The divertor power loads are investigated by scanning four quantities:

(i) plasma current $I_{P}$, from 130 to $340 \mathrm{kA}$

(ii) length of the outer divertor leg in the poloidal plane $L_{d i v}$, approximated by the distance of the $\mathrm{X}$-point from the vessel floor, from 21 to $64 \mathrm{~cm}$.

(iii) outer target flux expansion $f_{x}$, from 2 to 9 .

(iv) magnetic field direction, between forward field (FF, ion $\nabla B$ drift downwards, favorable for $\mathrm{H}-$ mode access) and reversed field (RF, ion $\nabla B$ drift upwards, unfavorable for H-mode access).

Each parameter scan is repeated for a range of values of the other parameters.

Divertor operational regime Langmuir probes (LPs) and a fast RCP are used to measure the variation of density, temperature and pressure in the SOL between 

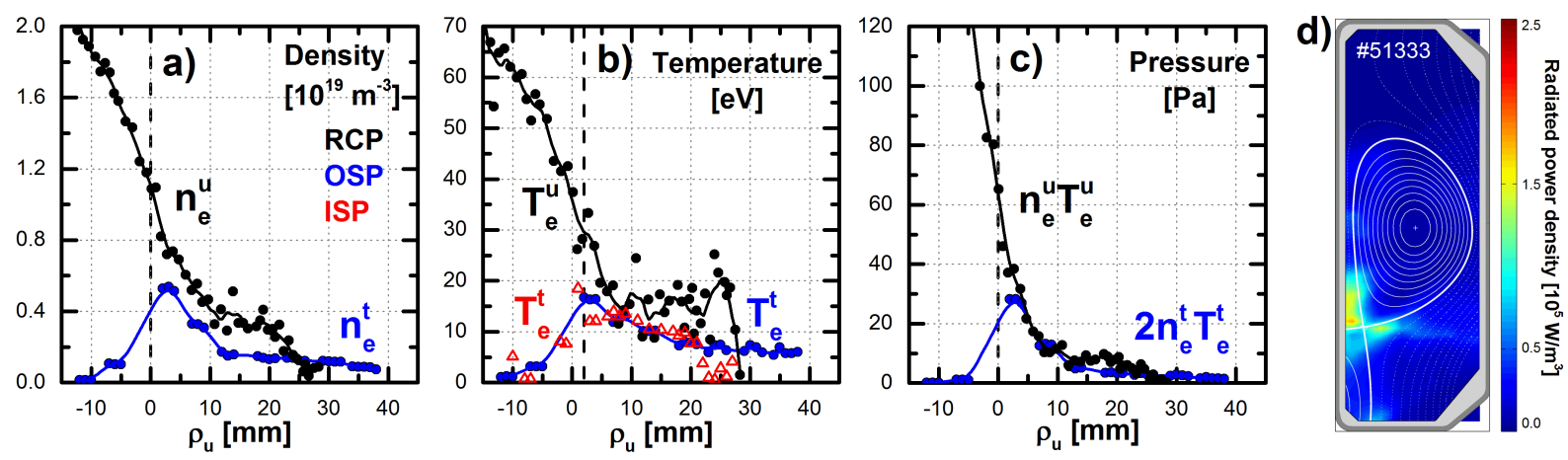

Figure 3. Density (a), temperature (b) and pressure (c) in the SOL, at the target (LPs) and at the mid-plane (RCP), for a typical shot of the database $\left(I_{P}=210 k A, L_{d i v}=36 \mathrm{~cm}, f_{x}=2.5\right.$ at OSP and $f_{x}=6$ at ISP, forward field).(d) For the same shot, radiated power density from a tomographic inversion of bolometric measurements.

the mid-plane and the divertor targets. For the inner strike point, only the temperature profile is presented, since some of the inner wall LPs do not yield reliable density and pressure measurements for these discharges [15]. Temperature and density gradients are seen along the magnetic field lines, figures $3 \mathrm{a}$ and $3 \mathrm{~b}$. The temperature at both targets, with peak values of $\approx 20 \mathrm{eV}$, implies that ionization will dominate over recombination processes. This is confirmed by the conservation of the total pressure (dynamic+static), figure 3c. In conclusion, both divertor legs are attached and in the high-recycling regime.

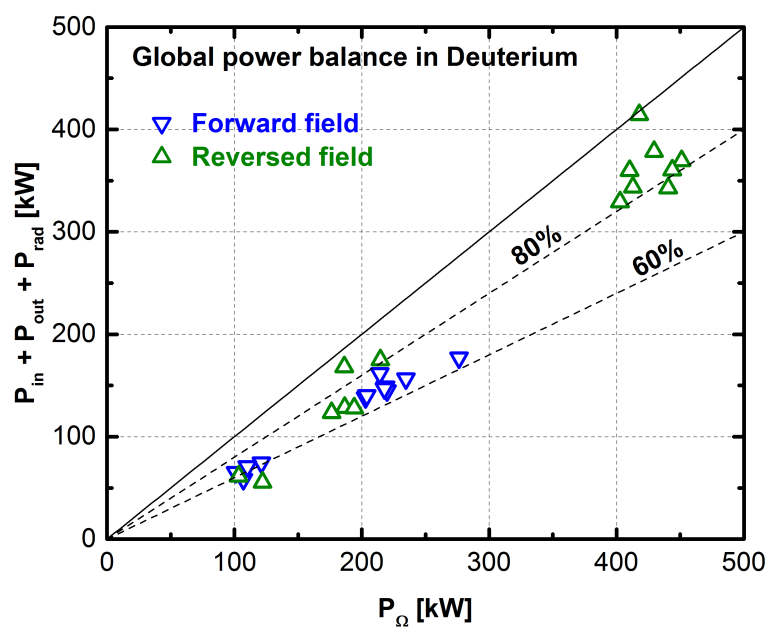

Figure 4. Power balance for the entire database of discharges: the measured exhaust power is 60 to $100 \%$ of the Ohmic heating power $P_{\Omega}$.

Global power balance The sum of the power measured at the targets with IR thermography $P_{\text {in }}+P_{\text {out }}$ and the total radiated power from bolometric inversions $P_{\text {rad }}$ corresponds to 60 to $100 \%$ of the Ohmic heating power, figure 4 , and is independent of the field direction. This systematic underestimation of the exhaust power can be partially ascribed to light reflection of the bolometer foils [16]. Target and radiated powers are averaged over the stationary phase of each discharge, which is typically 0.5 to $0.7 \mathrm{~s}$.

\section{Divertor heat flux parametric study}

This section presents the observed dependences of the in-out power ratio $P_{\text {in }} / P_{\text {out }}$, the SOL power fall-off length $\lambda_{q, u}$ and the divertor spreading factor $S_{u}$ (for both targets) on plasma current, outer divertor leg length, outer target flux expansion and magnetic field direction.

\subsection{Plasma current scan}

The plasma current $I_{P}$ is varied in forward field in a configuration with moderate outer leg length $L_{\text {div }}=36 \mathrm{~cm}$, flux expansion 6 at the ISP and 2.5 at the OSP. Here, the values of $f_{x}$ are averaged across the SOL in the vicinity of the separatrix from $\rho_{u}=[0$ to 2$] \mathrm{mm}$. The density is varied with $I_{P}$ to retain a Greenwald fraction $f_{\mathrm{GW}} \approx 0.25$. The power measured at both targets increases with $I_{P}$ as the Ohmic heating power also increases with $I_{P}$, figure 5a. The power fall-off length $\lambda_{q, u}$ decreases with increasing $I_{P}$, figure $5 \mathrm{~b}$, in qualitative agreement with observations on other tokamaks $[2,17]$. The exponents of -0.73 and -0.67 for the OSP and the ISP are similar, but $\lambda_{q, u}$ is somewhat larger at the OSP. The divertor spreading factor $S_{u}$ shows no clear dependence on $I_{P}$, but is systematically smaller at the inner than at the outer target, figure 5c. In contrast, the divertor spreading factor evaluated at the target, $S=S_{u} f_{x}$, is similar between inner and outer divertor, indicating that the $S_{u}$ asymmetry is mostly explained by the diverse target flux expansion. 

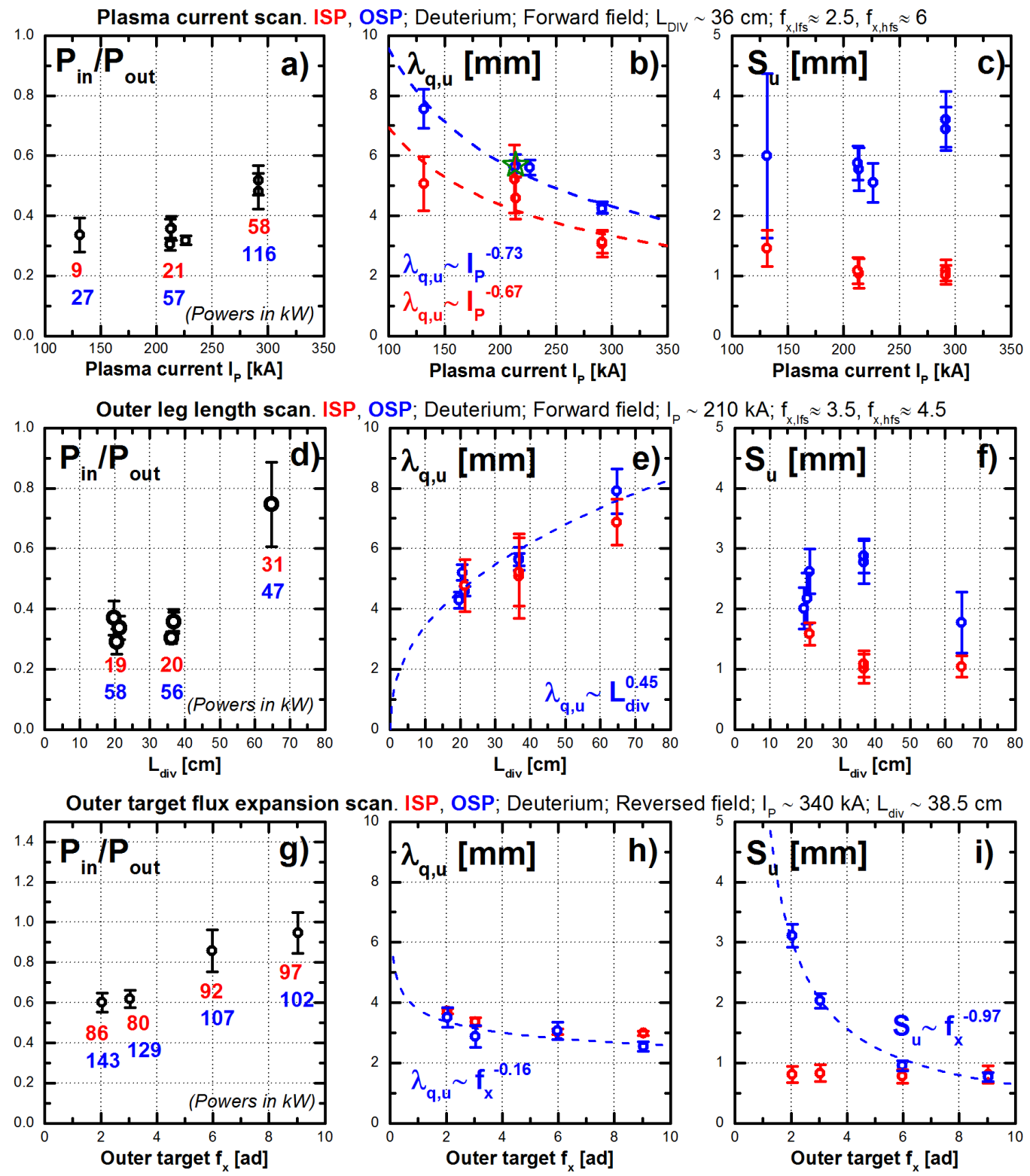

Figure 5. Power in-out ratio, SOL fall-off length and divertor spreading factor as a function of plasma current (a-b-c), outer leg length (d-e-f) and outer target flux expansion (g-h-i). The green star indicates the 2PM expectation for $\lambda_{q, u}$ from the upstream electron temperature profile (see section 4.3). The dashed lines correspond to power law fits.

\subsection{Outer divertor leg length scan}

With the elongated vessel of TCV, it is possible to move the plasma vertically while keeping shape and core properties constant. Three values of $L_{\text {div }}$ are investigated: $21 \mathrm{~cm}, 36 \mathrm{~cm}$ and $64 \mathrm{~cm}$, figure 6 , with $I_{P}=210 k A$, forward field and $f_{x}=4.5$ at the ISP and 3.5 at the OSP. The variation of $L_{d i v}$ changes the outer SOL parallel connection length $L_{\|}$, defined as the length of a magnetic field line connecting the OMP to the outer target. The parallel connection length is not constant in the SOL but diverges approximately logarithmically at the separatrix (figure $6 \mathrm{~b}, \mathrm{c}$ ). As the plasma is moved upwards, the connection length to the outer target increases uniformly over the entire SOL (figure 6c) while the connection length to the inner target remains constant (figure 6b). An inout power asymmetry increases for the longest outer divertor leg length, with less power flowing to the OSP and more to the ISP, figure $5 \mathrm{~d}$. The Ohmic power, the total radiated power and the total power to the targets, $P_{\text {in }}+P_{\text {out }}$, are constant through the scan. The outer divertor leg length also increases $\lambda_{q, u}$ at both targets, $\lambda_{q, u} \propto L_{\text {div }}^{0.45}$ (figure 5e), but does not affect $S_{u}$ (figure 5f), which is consistent with the LP analysis of this experiment [18]. These TCV experiments show 


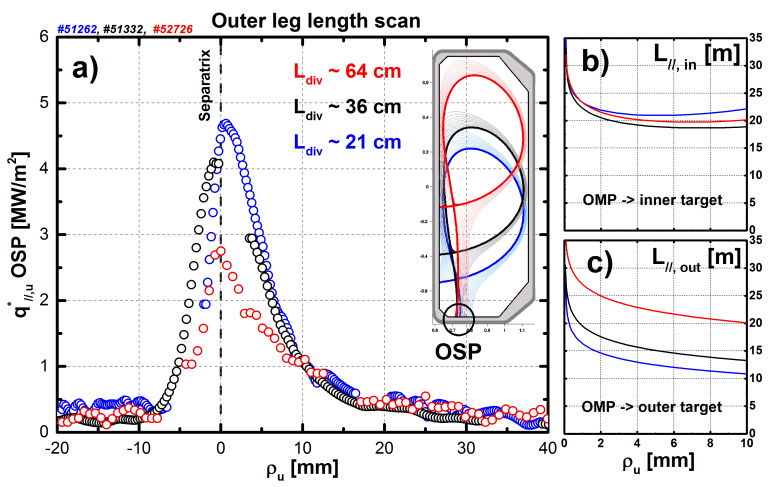

Figure 6. (a) Parallel heat flux profile mapped at the OMP for three values of $L_{d i v}$; an inlet shows the corresponding magnetic equilibria. (b),(c) Parallel connection length profiles for the inner and outer divertor legs.

a counter-intuitive dependence of the SOL power falloff length rather than of the divertor spreading on the divertor geometry.

\subsection{Flux expansion scan}

The flux expansion $f_{x}$ at the outer target is varied in reversed field discharges with $I_{P}=340 \mathrm{kA}$ and $L_{d i v}=38.5 \mathrm{~cm}$. Flux expansion affects the in-out power asymmetry (figure $5 \mathrm{~g}$ ) with increasing $f_{x}$ leading to an increase of the power at the ISP and a decrease at the OSP. The value of $\lambda_{q, u}$ for both targets weakly decreases for higher $f_{x}$, figure $5 \mathrm{~h}$. The value of $S_{u}$ at the inner target is constant but varies approximately as $S_{u} \propto f_{x}^{-1}$ at the outer target, figure 5i. The factor $f_{x}^{-1}$ corresponds to the compression of the heat flux profile when mapped upstream and the divertor spreading factor at the target (but perpendicular to the flux surfaces) $S=S_{u} f_{x}$ is consequently independent of the flux expansion.

\subsection{Magnetic field reversal}

The in-out power ratio weakly increases with a reversed magnetic field direction. The absolute values of $\lambda_{q, u}$ and the strength of the $\lambda_{q, u}$ variations change with magnetic field direction (compare figures $7 \mathrm{~b}$ and $7 \mathrm{e}$ with figures $5 \mathrm{~b}$ and $5 \mathrm{e})$. In reversed field, $\lambda_{q, \text { in }} \equiv \lambda_{q, u, \text { in }}$ is broader while $\lambda_{q, \text { out }} \equiv \lambda_{q, u, \text { out }}$ is narrower, such that $\lambda_{q, \text { in }} / \lambda_{q, \text { out }}<1$ in forward field and $\lambda_{q, \text { in }} / \lambda_{q, \text { out }}>1$ in reversed field. For the OSP, the dependence of $\lambda_{q, u}$ on plasma current and the outer divertor leg length are stronger in forward field. In contrast, the dependence of $\lambda_{q, u}$ at the ISP on the plasma current is stronger in reversed field. The value of $S_{u}$ at the OSP is smaller in reversed field, whereas $S_{u}$ at the ISP shows no variation (compare figures $7 \mathrm{c}$ and $7 \mathrm{f}$ with figures $5 \mathrm{c}$ and $5 \mathrm{f}$ ). Comparing the values of $S$ at the target again decreases the differences between the inner and outer targets as well as between the forward and reverse field directions.

\subsection{Summary}

In summary, the main experimental observations are:

(i) In-out power asymmetry $P_{\text {in }} / P_{\text {out }}$. The ratio $P_{\text {in }} / P_{\text {out }}$ increases from $\approx 0.3$ to $\approx 1.0$ with the outer divertor leg length and target flux expansion. Although slightly higher for reversed magnetic field, it is relatively independent of the plasma current.

(ii) SOL power fall-off length $\lambda_{q, u}$. For both targets, $\lambda_{q, u}$ decreases with higher plasma current, increases with longer outer divertor leg length and decreases weakly with higher outer target flux expansion. $\lambda_{q, u}$ measured at the inner and outer target is different and depends on the magnetic field direction, with $\lambda_{q, \text { in }} / \lambda_{q, \text { out }}<1$ in forward field while $\lambda_{q, \text { in }} / \lambda_{q, \text { out }}>1$ in reversed field. For the OSP, $\lambda_{q, u}$ variations with the plasma current and with the outer divertor leg length are stronger in forward field. In contrast, the variation of $\lambda_{q, u}$ with the plasma current at the ISP is stronger in reversed field.

(iii) Divertor spreading $S_{u}$. For both targets, $S_{u}$ does not show a clear dependence on plasma current nor leg length nor magnetic field direction. At the OSP only, $S_{u}$ manifests a strong inverse dependence on $f_{x}$. The divertor spreading factor evaluated at the target, $S=S_{u} f_{x}$, is independent of flux expansion and of the position of the strike point, as well as of plasma current, leg length and field direction.

\section{Result interpretation}

This section discusses a list of physical effects which may explain the observed dependencies of the in-out power asymmetry, SOL width and divertor spreading factor on $I_{P}, L_{d i v}, f_{x}$ and field direction, with continuous comparison to the TCV experimental data to verify whether these effects are plausible.

\subsection{Analytic model for the SOL}

An analytic model for the SOL is used to evaluate the extent to which basic physics and geometry can explain the observed dependences. Electron heat conduction is taken to be the dominant mechanism for heat transport from the outer mid-plane along the field lines to the targets, following the main hypothesis of the Two-Point Model (2PM) [19]. Any cross-field transport is assumed to be driven by the temperature gradient and to occur mainly in the upstream SOL 

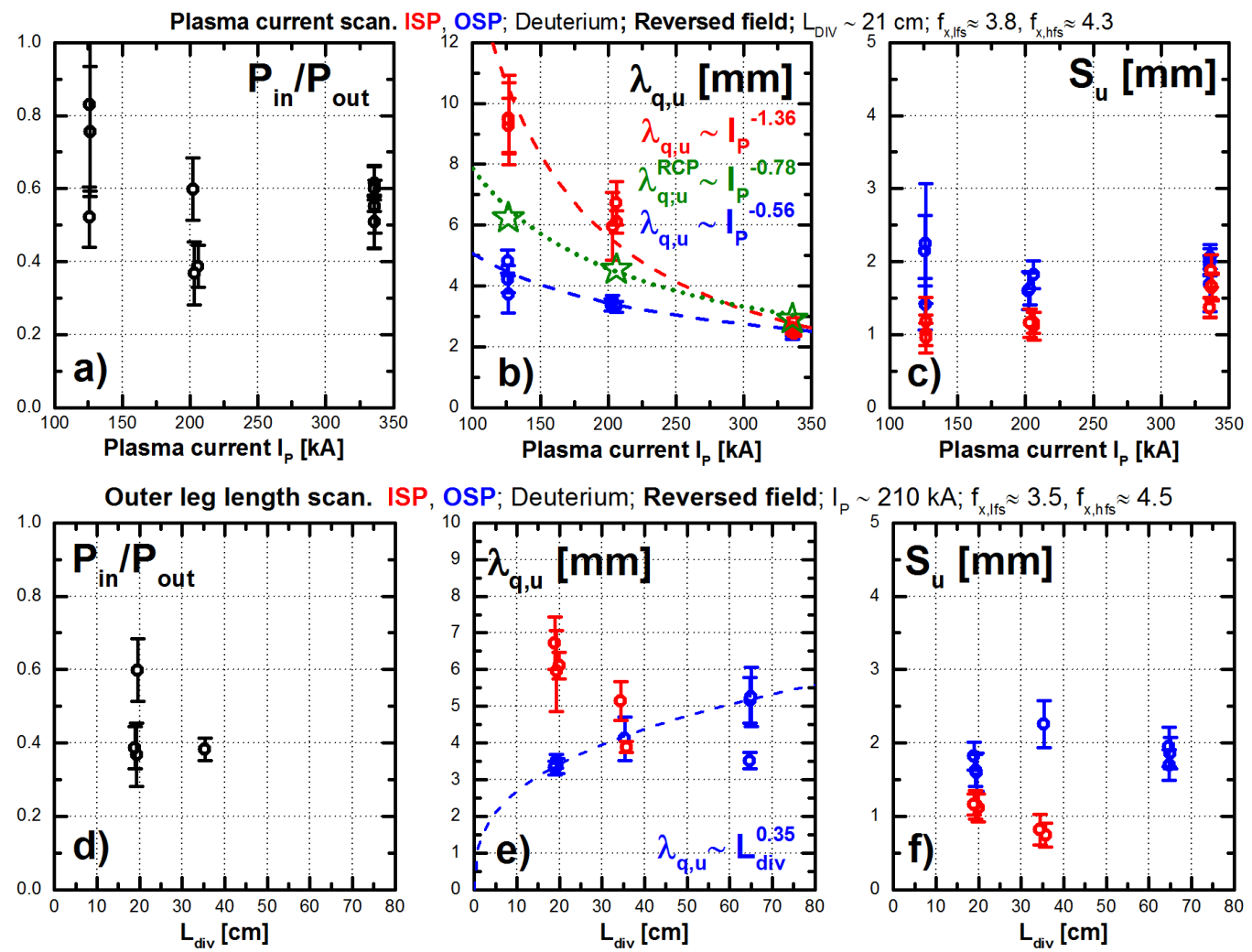

Figure 7. $P_{\text {in }} / P_{\text {out }}, \lambda_{q, u}$ and $S_{u}$ for plasma current scan (a-b-c) and divertor leg length scan (d-e-f) with reversed magnetic field. Green stars indicate the estimates $\lambda_{q, u}^{R C P}$ from the upstream electron temperature profile measured with the RCP (see section 4.3).

surrounding the confined plasma. The model adopts substantial approximations, such as no sources or sinks of heat and particles or convective contribution to the heat transport. The derivation of the model is discussed in detail in Appendix 8.1. The model predicts an in-out power ratio

$P_{\text {in }} / P_{\text {out }}=L_{\|, \text {out }} / L_{\|, \text {in }}$

where $L_{\|, \text {out }}$ and $L_{\|, \text {in }}$ are the parallel connection lengths to the inner and to the outer targets respectively. Note that $L_{\|, \text {out }}$ and $L_{\|, \text {in }}$ are assumed to be uniform across the SOL. According to equation 3 , increasing the connection length to one target results in a redistribution of power to the other target. This redistribution from the target with the longer connection length is caused by the decrease in the parallel temperature gradient, which reduces the conductive heat flux to that target.

This analysis then extends the 2PM to the cross-field direction, to examine how the fall-off length of the heat flux profile at the OMP may be affected by plasma or divertor parameters. A simple power balance holds between the heat deposited at the targets and the radial derivative of the power flux across flux surfaces. Our model generates a second order partial differential equation in $q_{\|, u, \text { out }}$ or alternatively in $q_{\|, u, \text { in }}$, the upstream parallel heat flux profiles flowing to the outer and to the inner target, respectively. The solution profiles, parameterised as decaying exponentials, differ only by a numerical factor that reflects the power asymmetry of equation 3 , and have the same decay length,

$$
\begin{aligned}
\lambda_{q} \propto & L_{\|, \text {out }}^{2 / 9}\left(1+\frac{L_{\|, \text {out }}}{L_{\|, \text {in }}}\right)^{-2 / 9} \chi_{\perp}^{7 / 9} n_{e, u}^{7 / 9} \\
& \times P_{\text {sep }}^{-5 / 9} I_{P}^{-2 / 9} B_{\text {tot }, u}^{2 / 9} a R_{u}^{5 / 9}
\end{aligned}
$$

where $n_{e, u}, B_{t o t, u}$ and $R_{u}$ are the plasma density, total magnetic field and major radius at the OMP, $\chi_{\perp}$ the perpendicular transport coefficient and $a$ the plasma minor radius. Equation 4 can be adjusted by using a Bohm-like diffusivity for drift waves, $\chi_{\perp} \propto T_{u, s e p} / B_{\text {tot }, u}$, or ballooning modes, $\chi_{\perp} \propto\left(T_{u, s e p} q\right) / B_{t o t, u}$, see Appendix 8.1.

For model to experiment comparison, an effective connection length for each target must be chosen. A connection length profile, averaged across the SOL in

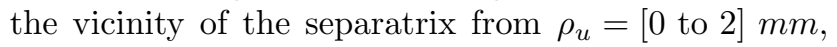
was used. In the following, this effective connection length is simply referred to as $L_{\|}$(or more explicitly as $L_{\|, \text {out }}$ and $L_{\|, \text {in }}$ when required).

For the outer divertor leg length scan in TCV and the values of $L_{\|, \text {in }}$ and $L_{\|, \text {out }}$ obtained, equation 4 can be approximated by $\lambda_{q, u} \propto L_{\|, \text {out }}^{0.13}$ (fixed $\chi_{\perp}$ ), 
$\lambda_{q, u} \propto L_{\|, \text {out }}^{0.21}$ (Bohm-like diffusivity for drift waves or ballooning modes).

The $I_{P}$ dependencies from the other parameters of equation 4 must be accounted for before seeking any further plasma current scaling. With $L_{\|, \text {out }} \propto I_{P}^{-1}$, $L_{\|, \text {in }} \propto I_{P}^{-1}, n_{e, u} \propto I_{P}$ (constant Greenwald fraction) and $P_{\text {sep }} \propto P_{\Omega} \propto I_{P}^{1.6}$ (estimated from TCV database of Ohmic plasmas), Equation 4 shows $\lambda_{q, u}$ scalings with the plasma current: $\lambda_{q, u} \propto I_{P}^{-0.56}$ (fixed $\chi_{\perp}$ ), $\lambda_{q, u} \propto I_{P}^{-0.53}$ (drift waves), $\lambda_{q, u} \propto I_{P}^{-1.16}$ (ballooning modes).

\subsection{In-out power asymmetry}

The observed ratio $P_{\text {in }} / P_{\text {out }}$ depends linearly on the inverse ratio of the connection lengths $L_{\|, \text {out }} / L_{\|, \text {in }}$ in both the $L_{d i v}$ and $f_{x}$ scans (figure $8 \mathrm{a}$ and $8 \mathrm{c}$ ), as predicted by the model, equation 3. Quantitatively, the observed $P_{\text {in }} / P_{\text {out }}$ has an offset with respect to the exact ratio, with the power measured at the inner target being systematically less (or at the outer target systematically more) than expected. The inout power asymmetry does not manifest any significant dependence on plasma current, both in forward and reverse field. This is also consistent with the model, since the plasma current does not affect the ratio $L_{\|, \text {out }} / L_{\|, \text {in }}$. Such evidence is in contrast with previous findings on JET [20] and JT-60U [21, 22], where, for low-density L-mode NBI-heated discharges, the in-out power asymmetry was observed to depend on the edge safety factor $q_{95}$, and, therefore, on the plasma current. $P_{\text {in }} / P_{\text {out }}$ weakly increases for a reversed magnetic field direction. This behavior is consistent with observations on JET [20, 23], DIII-D [24], Alcator C-Mod [25] and JT-60U [21, 22].

\subsection{SOL power fall-off length $\lambda_{q, u}$}

Comparison with conductive SOL model The observed dependence of $\lambda_{q, u}$ on plasma current depends on the SP and magnetic field direction, with the regression exponent ranging between -0.56 and -1.36 , see figures $5 \mathrm{~b}$ and $7 \mathrm{~b}$ and table 1 . The model predicts a range of similar exponents for the various transport models, with the drift waves being the weakest (-0.53) and the ballooning modes the strongest dependence (-1.16), but independent of strike point location and field direction. While the strength of the $I_{P}$ dependence is within the predictions of the considered transport models, the observed variations among strike points and field directions must result from physics that is not included in the model. The predicted variation of $\lambda_{q, u}$ with $L_{\|, \text {out }}$, $\lambda_{q, u} \propto L_{\|, \text {out }}^{0.13} / L_{\|, \text {out }}^{0.21}$ for fixed and Bohm-like $\chi_{\perp}$ respectively, is too weak to explain the $L_{\text {div }}$ scan and is the wrong direction for the flux expansion scan. In conclusion, while the conductive model may describe the gen- eral $I_{P}$ dependence, it does clearly not include enough physics to describe the observed variations of $\lambda_{q, u}$ with SP location, field direction and geometric variations of the divertor.

\begin{tabular}{c|c|c}
\hline & Forward field & Reversed field \\
\hline OSP & $\lambda_{q, u} \sim L_{\|, \text {out }}^{0.92}\left(L_{\text {div }}\right)$ & $\lambda_{q, u} \sim L_{\|, \text {out }}^{0.40}\left(L_{\text {div }}\right)$ \\
& $\lambda_{q, u} \sim L_{\|, \text {out }}^{-0.14}\left(f_{x}\right)$ & $\lambda_{q, u} \sim L_{\|, \text {out }}^{-0.57}\left(f_{x}\right)$ \\
& $\lambda_{q, u} \sim I_{P}^{-0.73}$ & $\lambda_{q, u} \sim I_{P}^{-0.56}$ \\
\hline ISP & $\lambda_{q, u} \sim L_{\|, \text {out }}^{-0.10}\left(f_{x}\right)$ & $\lambda_{q, u} \sim L_{\|, \text {out }}^{-0.52}\left(f_{x}\right)$ \\
& $\lambda_{q, u} \sim I_{P}^{-0.67}$ & $\lambda_{q, u} \sim I_{P}^{-1.36}$ \\
\hline
\end{tabular}

Table 1. Power laws for measured $\lambda_{q, u}$ variation with $L_{\|, \text {out }}$ and $I_{P}$ change between inner (ISP) and outer (OSP) strike point and with magnetic field reversal. The parameter varied in the scan is shown in brackets.

Comparison with prediction from $R C P$ The assumption that parallel transport in the SOL is described by the $2 \mathrm{PM}$ in the conduction limited regime yields, using equation 11 in Appendix 8.1, a simple relation between $\lambda_{q, u}$ and the decay length of the upstream electron temperature in the SOL: $\lambda_{q, u}^{R C P}=2 / 7 \cdot \lambda_{T_{e}, u}$. The RCP provides an estimate of $\lambda_{T_{e}, u}$ so that $\lambda_{q, u}^{R C P}$ can be computed and compared to the value estimated by IR thermography at the divertor target. The RCP was routinely used in the plasma current scan in reversed field, figure $7 \mathrm{~b}$, where its estimate of $\lambda_{q, u}^{R C P}$ lies between the values derived from inner and outer target measurements. The difference decreases with increasing $I_{P}$ vanishing at the highest value, where $\lambda_{q, i n}, \lambda_{q, \text { out }}$ and $\lambda_{q, u}^{R C P}$ agree. In conclusion, the RCP measurements are consistent with the hypothesis that additional physics causes the in-out $\lambda_{q, u}$ asymmetry and suggest that this physics is less important at higher plasma currents.

Role of $\nabla B$ and curvature $B$ drifts $\nabla B$ and curvature $B$ drifts transfer plasma across the separatrix into the SOL. In forward field, these drifts are directed towards the active X-point, i.e. downwards, and therefore broaden the outer SOL while narrowing the inner SOL. In reversed field, these drifts are directed upwards, causing a broadening of the inner SOL and a narrowing of the outer SOL. The $\nabla B$ and curvature $B$ drifts are therefore prime candidates to explain the observed in-out $\lambda_{q, u}$ asymmetry and dependence on magnetic field direction. A higher plasma current reduces the parallel connection length from the OMP to the X-point for both inner and outer SOL. This enhances parallel transport, by increasing the parallel temperature gradient, and, thereby, weakens any impact of the drifts on $\lambda_{q, u}$ (i.e. the $\lambda_{q, u}$ in-out asymmetry, figure $7 \mathrm{~b}$ ). The effect of these drifts should be independent of the outer divertor leg length, since 

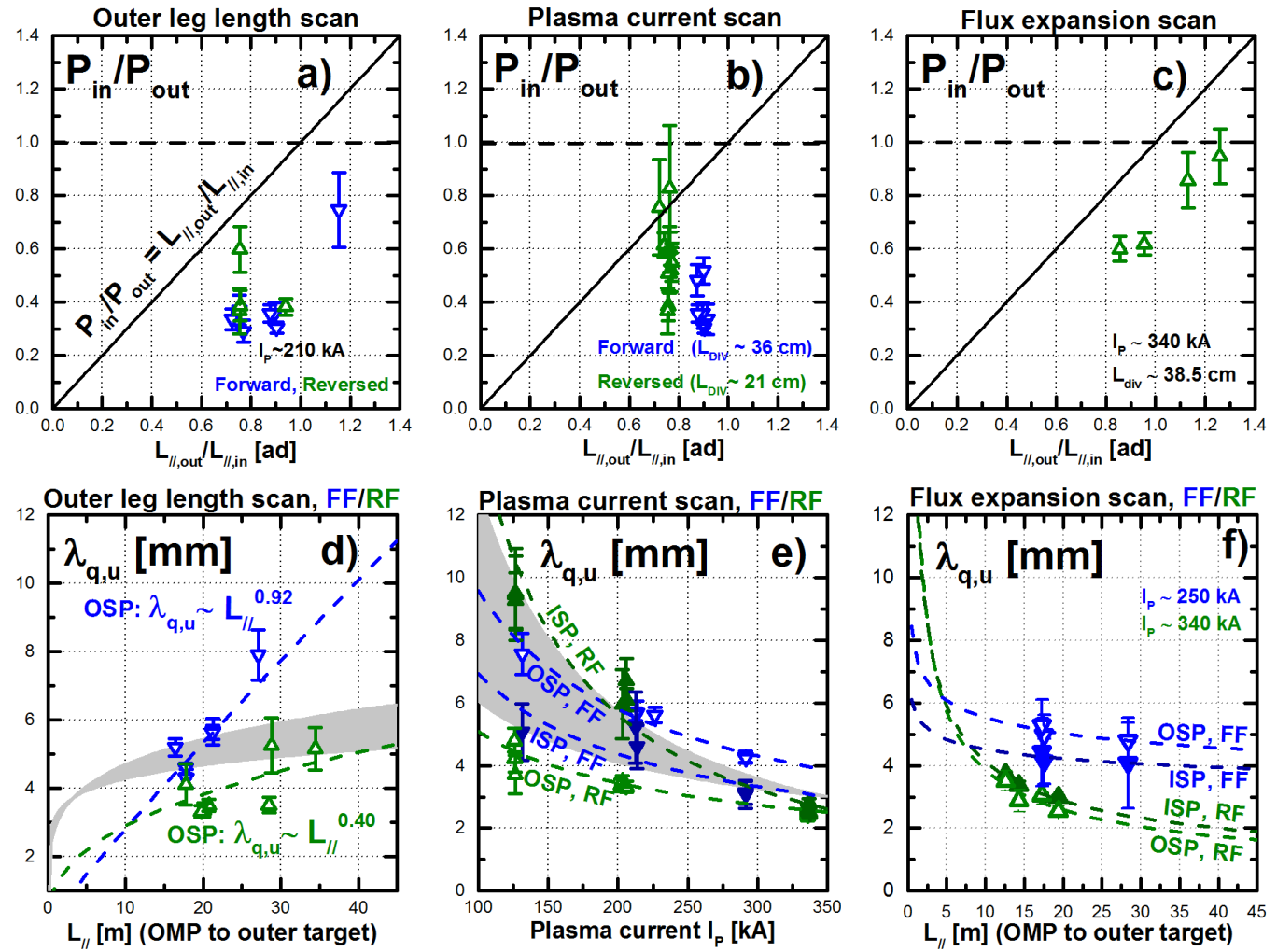

Figure 8. Test of model predictions for $P_{\text {in }} / P_{\text {out }}$ and $\lambda_{q, u}$ when connection length is changed via $L_{d i v}$ (a-d), $I_{P}$ (b-e) or $f_{x}$ (c-f). Blue diamonds pointing down/green diamonds pointing up represent forward/reversed field. Filled/empty symbols for ISP/OSP data. The predicted trends for $\lambda_{q, u}$ are in light gray.

the outer divertor leg flux surfaces are approximately vertical. Therefore, these drifts cannot explain the observed $\lambda_{q, u}$ broadening with $L_{d i v}$.

Role of $E \times B$ drifts The $E \times B$ drifts can have an important role in the transport of particles in the divertor region $[20,25]$. Simulations with the two dimensional edge plasma transport code UEDGE were recently carried out for two TCV discharges included in this study $\left(I_{P}=210 \mathrm{kA}, L_{d i v}=21 \mathrm{~cm}, f_{x}=4.5\right.$ at ISP and 3.5 at OSP, forward and reversed field) [26]. The simulations show that, for both forward and reversed field, along most of the outer divertor leg the radial component of the $E \times B$ drift carries particles from the separatrix towards the far SOL. During a $L_{d i v}$ scan, such a mechanism may cause a broadening of the outer SOL $\lambda_{q, u}$, independently of the field direction, which would increase the broadening predicted by the analytic model. With a higher $f_{x}$, one can expect a higher poloidal component of parallel gradients along field lines of the outer SOL due to the decreasing pitch angle of the field lines. This causes an increase of the radial $E \times B$ drift velocity in the outer divertor leg that competes with the increased distance between flux surfaces. Quantitative studies are required to determine whether the radial drift velocity changes faster than the spacing between flux surfaces. If the latter dominates, the effect of $E \times B$ drifts on the SOL width would be less important for higher flux expansions, consistent with the observed weak decrease of $\lambda_{q, u}$ with $f_{x}$ at the OSP, figure $5 \mathrm{~h}$.

Asymmetric turbulent transport in the divertor The distinct dependence of $\lambda_{q, u}$ on $L_{\text {div }}$ may be explained by the turbulent perpendicular transport in the divertor leg towards the common flux region being stronger than towards the private flux region. Such an asymmetric cross-field transport along the divertor leg would increase $\lambda_{q, u}$ rather than $S_{u}$ as observed in the TCV experiments. Recent simulations [27] with the 3D fluid turbulent code TOKAM3X [28] of some plasma pulses of this paper $\left(L_{d i v}\right.$ scan with $I_{P}=210 k A$ in forward field) confirm the validity of this hypothesis. Since the decay length $\lambda_{q, u}$ captures, by construction, any asymmetric component of the cross-field transport in the divertor, the classical interpretation of $\lambda_{q, u}$ as a pure main SOL quantity is no longer valid in the presence of asymmetric transport in the divertor. To decouple the contributions to $\lambda_{q, u}$ of the upstream and divertor transport, the model suggested by [2] 
requires extension, for instance, by adding a divertor contribution to $\lambda_{q, u}$ which could be proportional to the divertor depth $L_{d i v}$.

\subsection{Divertor spreading factor}

Diffusive model The spreading factor at the target $S$ captures the isotropic cross-field diffusion of heat and particles into the SOL and into the private flux region between the X-point and the targets. This process can be seen as a competition between parallel and perpendicular heat diffusion [29]:

$S=l_{\mathrm{x}} \sqrt{\frac{\chi_{\perp}}{\chi_{\|}}}=l_{\mathrm{x}} T_{e}^{-5 / 4} n_{e}^{1 / 2} \sqrt{\frac{\chi_{\perp}}{\kappa_{0}}}$

where $l_{\mathrm{X}}$ is the parallel connection length between the $\mathrm{X}$-point and target, $\chi_{\perp}$ and $\chi_{\|}$the heat diffusivities, $n_{e}$ and $T_{e}$ the electron density and temperature, with the parallel heat transport described by electron SpitzerHarm diffusivity [30] $\chi_{\|}=\kappa_{0} T_{e}^{5 / 2} / n_{e}$. With a strong temperature dependence of the parallel diffusivity $\chi_{\|}$, the perpendicular diffusion is only relevant for sufficiently low temperatures. In attached conditions, the divertor temperature is too high for any significant cross-field spreading. In the proximity of the divertor target, the heat diffuses across flux surfaces and $S$ increases. This suggests an integral expression for $S$ [31], which yields (see Appendix 8.2):

$S=2 \sqrt{\frac{\chi_{\perp} n_{\mathrm{x}}}{\kappa_{0}}} T_{\mathrm{x}}^{-5 / 4} l_{\mathrm{x}}$

where $T_{\mathrm{X}}$ and $l_{\mathrm{x}}$ are the $\mathrm{X}$-point temperature and density.

Divertor spreading at the outer target The divertor spreading factor at the target, $S=S_{u} f_{x}$ is observed to be independent of plasma current, outer divertor leg length, outer target flux expansion and magnetic field direction. According to the diffusive model, equation 5, when increasing the plasma current at fixed Greenwald fraction, the increase in the X-point density can balance the decrease in $l_{\mathrm{x}}$ and a potential increase in $T_{\mathrm{x}}$, resulting in no variation of $S$. When the parallel connection length is increased via a longer outer divertor leg length or a higher outer target flux expansion, the $\mathrm{X}$-point temperature weakly increases since, according to the $2 \mathrm{PM}, T_{u} \sim L_{\|}^{2 / 7}$ for constant $q_{\|}$. According to the diffusive model, equation 5, the competition between a longer $l_{\mathrm{x}}$ and a higher $T_{\mathrm{X}}$ weakens the variation of $S$.

Correlation with target electron temperature A previous study in ASDEX Upgrade (AUG) found a correlation of $S$ with the electron temperature at the target [32]. On TCV, the electron temperature at the target $T_{e, t}$ does not change significantly during the scan of the

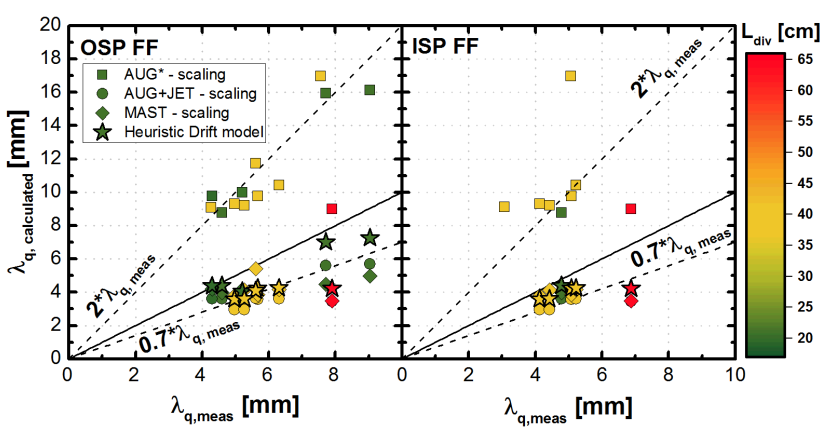

Figure 9. Measured target $\lambda_{q, u}$, for inner (ISP) and outer (OSP) strike point, in forward magnetic field, compared to the prediction by the scaling law from AUG [29] (squares, regression modified as in [33]), AUG+JET [34] (circles) and MAST [35] (diamonds), and to the prediction from the drift model of [36].

plasma current and of the outer target flux expansion. In the $L_{\text {div }}$ scan, despite $T_{e, t}$ variations of up to $40 \%$, from a peak value of $20 \mathrm{eV}$ to $12 \mathrm{eV}$, no change in $S$ is measured. This differs from the inverse dependence of the outer divertor spreading factor on $T_{e, t}$ observed in [32].

\section{Comparison with previous studies of $\lambda_{q}$}

The study performed on TCV extends the range of geometric variations and adds new dimensions. The variation of the outer divertor leg length has revealed a clear and unexpected effect of divertor geometry modifications on the SOL power fall-off length $\lambda_{q, u}$.

\subsection{Similar observations in DIII-D}

Some evidence of a similar effect of the divertor leg length on SOL transport has been reported from the DIII-D tokamak [37]. Measurements of the outer target heat flux profile for L-mode attached SN plasmas with drift towards the active X-point show a broadening of the target profile and a slight decrease in integrated power with increased leg length and a simultaneously decreased flux expansion.

\subsection{L-mode scaling laws}

A recent study in AUG [29] proposes a scaling of $\lambda_{q, u}$ measured at the outer divertor target of attached L-mode SN plasmas, with the ion $\nabla B$ direction towards the active $\mathrm{X}$-point (i.e. forward field):

$\lambda_{q, u}^{\mathrm{AUG}}=0.15 B_{\text {pol,u }}^{-0.62} A^{-0.15} n_{\text {edge }}^{0.94} W_{\mathrm{MHD}}^{-0.99}$

with $B_{p o l, u}$ the poloidal magnetic field at the OMP, $A$ the main ion mass number, $n_{\text {edge }}$ the edge electron density and $W_{\text {MHD }}$ the plasma stored energy. This scaling may be generalized to other tokamaks by replacing the stored energy term $W_{\text {MHD }}$ with $W_{\mathrm{MHD}} V_{\mathrm{AUG}} / V$, where $V$ is the plasma volume 


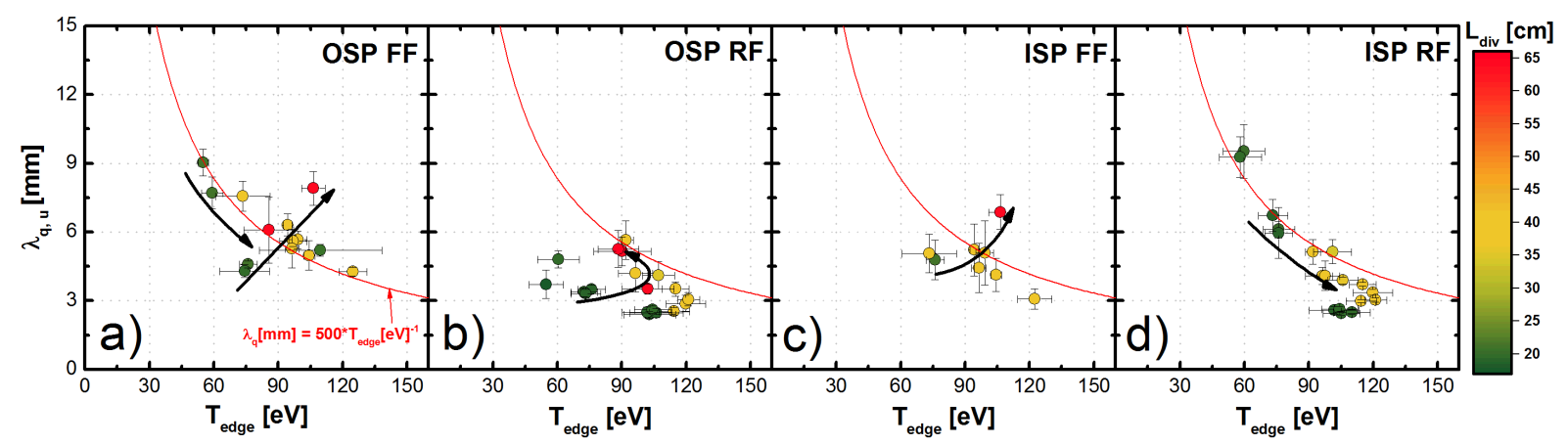

Figure 10. Measured target $\lambda_{q, u}$, for inner (ISP) and outer (OSP) strike point, forward (FF) and reversed (RF) magnetic field, as a function of edge electron density $T_{e d g e}$. A trend line $\lambda_{q, u} \propto T_{\text {edge }}^{-1}$ is also plotted. The arrows indicate the $L_{d i v}$ scans at fixed $I_{P}$ and $f_{x}$.

[33]. For the TCV plasmas discussed in this paper, $V \approx 1.35 \mathrm{~m}^{3}$ and $A=2$. The $n_{\text {edge }}$ is obtained from Thomson Scattering core density profiles evaluated at normalized poloidal flux coordinate $\rho=0.95$. It is found that the scaling exceeds the TCV measurements, except for the longest divertor leg, by a factor of approximately 2 , figure 9 .

The scaling for $\lambda_{q, u}$ obtained from AUG+JET data (equation (6) of [34], $H_{2}$ and $D_{2}$ plasmas) or MAST data (equation (4) of [35], Double Null Divertor) are found to be in better agreement with the TCV measurements, figure 9. For a plasma current scan at fixed Greenwald fraction in TCV, $q_{95} \propto B_{\text {pol,u }}^{-1} \propto I_{P}^{-1}$, $P_{\mathrm{SOL}} \propto P_{\Omega} \propto I_{P}^{1.6}$ and $\bar{n}_{e} \propto I_{P}$, where $\bar{n}_{e}$ is the lineaveraged density and $P_{\mathrm{SOL}}=P_{\Omega}-P_{\text {rad,core }}$ is the power crossing the separatrix. Therefore, the AUG + JET scaling law of [34] yields $\lambda_{q, u}^{\text {AUG }+ \text { JET }} \propto I_{P}^{-0.788}$ for the TCV experiments, similar to the dependence observed at TCV in forward field, table 1 . The scaling from MAST gives a weaker variation, $\lambda_{q, u}^{\mathrm{MAST}} \propto I_{P}^{-0.304}$. The ratio $W_{\text {MHD }} / n_{\text {edge }}$, equation 6 , is interpreted in [29] as a proxy for the edge electron temperature $T_{\text {edge }}$, implying $\lambda_{q, u} \propto T_{\text {edge }}^{-1}$. For the plasmas discussed in this paper, such scaling with $T_{\text {edge }}$ captures the $I_{P}$ dependence for both divertor targets and magnetic field directions, figure 10. It does not, however, capture the $L_{d i v}$ dependence, indicating that the parameterisation of equation 6 is not able to describe the observed $\lambda_{q, u}$ increase for longer outer divertor legs.

\subsection{Multi-machine H-mode scalings}

The dependence of $\lambda_{q, u}$ on the divertor leg length is also not included in the current multi-machine scalings for H-mode Single Null plasmas [2]. Here the scalings were reported on the heat flux width as measured at the OSP in forward field. It is found that the TCV data from $I_{P}$ and $L_{d i v}$ scans in forward field agree qualitatively with the regression \# 14 from [2], figure 11. The dependence of $\lambda_{q, u}$ on the divertor leg length,

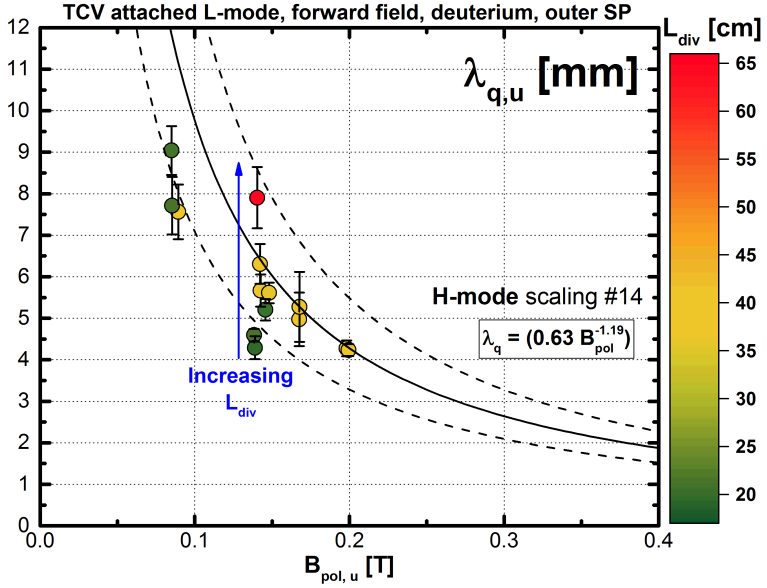

Figure 11. Outer target $\lambda_{q, u}$ as a function of $B_{p o l, u}$ in forward field, compared to multi-machine H-mode regression \#14 (black line, dashed lines are the error bars) from [2].

observed at TCV, is within the uncertainty of the regression scalar coefficients. The values of $\lambda_{q, u}$ in TCV L-modes match the H-mode scaling. In ASDEX Upgrade, in contrast, $\lambda_{q, u}$ in L-mode was found to be approximately two times greater than in H-mode [29].

\subsection{Drift model}

In the heuristic drift-dominated model of [36], developed for the case of low gas-puff H-mode plasmas, the SOL width is determined by vertical $\nabla B$ and curvature B drifts in the SOL surrounding the confined plasma. The SOL width measured at the outer divertor of TCV matches the drift model prediction, equation (6) of [36], for the shortest outer divertor leg length, but exceeds the model expectations for increased leg length, figure 9. This is consistent with the hypothesis that the observed broadening of $\lambda_{q, u}$ with the leg length results from asymmetric turbulent transport in the divertor. 


\section{Conclusion}

The paper presents an investigation of the power loads at the inner and outer divertor targets of L-mode attached Single-Null deuterium plasmas at constant Greenwald fraction under variable divertor geometries in the TCV tokamak. The target heat flux profiles, measured with the Infrared Thermography system, are studied as a function of plasma current and of variations in the magnetic divertor geometry, namely changes of the length of the outer divertor leg and of the target flux expansion. The SOL width $\lambda_{q, u}$ and the divertor spreading factor $S_{u}$ are estimated from target heat flux parameterisation.

The in-out target power ratio $P_{\text {in }} / P_{\text {out }}$ is found to increase with divertor leg length and flux expansion, but is largely independent of the plasma current. Under the assumption of parallel heat transport in the SOL dominated by electron conduction, this is interpreted as a change in the power sharing between inner and outer divertors, caused by the variation of the flux tube length from the outer mid-plane to the outer target affecting the thermal conductance of the outer divertor.

The SOL width $\lambda_{q, u}$ measured at both targets is narrower for higher plasma currents. A simple analytic conductive model for the SOL, developed ad-hoc, yields a scaling of $\lambda_{q, u}$ with the plasma current, which is similar to these observations. The model can predict a range of exponents depending on the assumed crossfield transport model, with the drift waves yielding the weakest and the ballooning modes the strongest $I_{P}$ dependence.

The SOL width $\lambda_{q, u}$ is different for the inner and outer target and depends on the magnetic field direction. These observations are not captured by the simple model and may be the result of ion $\nabla B$ and curvature $B$ drifts in the SOL.

The SOL width $\lambda_{q, u}$ measured at the outer target is broader for longer outer divertor legs. The same analytic model predicts only a very weak increase of $\lambda_{q, u}$ for this case, with very little dependence on the assumed transport model. The variation of $\lambda_{q, u}$ with the leg length may be interpreted as asymmetric cross-field diffusion, as, for instance, expected from turbulence, along the divertor leg contributing primarily to a further broadening of $\lambda_{q, u}$ rather than a symmetric spreading through the divertor spreading factor.

The measured divertor spreading factor at the target (but perpendicular to the flux surfaces) $S=S_{u} f_{x}$ is found independent of plasma current, divertor leg length, flux expansion, position of the strike point and magnetic field direction. This is evidence that $S$ is only determined by the conditions at the target, which is consistent with the hypothesis that a significant fraction of the divertor transport is asymmetric and contributes to $\lambda_{q, u}$.

The TCV $\lambda_{q, u}$ values are moreover in reasonable agreement with the AUG+JET and the MAST L-mode scalings and also match, in absolute value, the latest multi-device H-mode scalings.

Possible implications for future devices A first possible implication concerns the measured broadening of the SOL $\lambda_{q, u}$ for longer divertor leg. If such broadening was experimentally observed also for $\mathrm{H}$-mode plasmas, a long-legged divertor for a future fusion device (e.g. DEMO) would become very beneficial in terms of power exhaust, with a broader SOL, and thus lower peak heat fluxes and presumably easier access to detachment, compared to present-day predictions. A long-legged divertor may represent a step towards the solution of the power exhaust issue but would concomitantly reduce the volume of the vessel available for the confined burning plasma, limiting the fusion power. A second possible implication for future fusion experiments regards the spreading factor $S$. The finding that $S$ does not change with flux expansion can be reasonably extended to $\mathrm{H}$-mode plasmas since the divertor conditions should be independent of the plasma confinement. Therefore, for a future fusion device, a divertor with low $f_{x}$ appears as the most advantageous choice as, compared to the high $f_{x}$ case, it allows for a greater tilt of the divertor target (assuming fixed the minimum grazing angle of the field lines) while benefiting from the same divertor heat flux spreading $S$ and, thereby, reducing the peak heat flux onto the target.

\section{Acknowledgments}

Very useful and constructive discussions with J. Boedo, N. Christen, B.P. Duval, M. Faitsch, O. Février, J. Harrison, F. Nespoli, P. Ricci, U. Sheikh, K. Verhaegh and all members of TCV boundary physics team are truly acknowledged. This work has been carried out within the framework of the EUROfusion Consortium and has received funding from the Euratom research and training programme 2014-2018 under grant agreement No 633053. The views and opinions expressed herein do not necessarily reflect those of the European Commission.

\section{Appendix}

\subsection{Analytic conductive SOL model}

The power crossing a flux surface in the SOL may be written as:

$P_{\perp}=-4 \pi^{2} R_{0} a \sqrt{\frac{1+\kappa^{2}}{2}} \chi_{\perp} n_{e} \frac{\partial T_{u}}{\partial \rho_{u}}$ 
where $R_{0}$ is the major radius, $a$ the minor radius, $k$ the elongation, $\chi_{\perp}$ the perpendicular heat diffusivity, $n_{e}$ the upstream density, $T_{u}$ the temperature in the SOL and $\rho_{u}$ the upstream coordinate. No hypothesis on the physics determining cross-field transport (classical, neoclassical or turbulent) is made here but the perpendicular heat diffusivity $\chi_{\perp}$ is taken to be constant. The power per flux tube at the inner (' $i$ ') and outer (' $O$ ') divertor target is:

$\frac{\partial P_{\perp}}{\partial \rho_{u}}=-2 \pi\left(R_{t, i} q_{p, t, i} f_{x, t, i}+R_{t, o} q_{p, t, o} f_{x, t, o}\right)$

The target flux expansion is defined as:

$f_{x, t}=\frac{R_{u} B_{p, u}}{R_{t} B_{p, t}}$

while the poloidal heat flux at the target $q_{p, t}$ is:

$q_{p, t}=\frac{B_{p, t}}{B_{\text {tot }, t}} q_{\|, t}=\frac{B_{p, t}}{B_{t o t, t}} \frac{B_{t o t, t}}{B_{t o t, u}} q_{\|, u}$

The $2 \mathrm{PM}$ is adopted to describe the transport parallel to the field lines in the conduction limited regime. Assuming an upstream temperature $T_{u}$ which is much larger than the plasma temperature at the target, $T_{u}$ depends only on the heat flux and the connection length,

$T_{u}=\left(\frac{7 q_{\|, u} L_{\|}}{2 \kappa_{0}}\right)^{2 / 7}$

Note that some of the plasmas discussed in this paper are found not to meet this condition, e.g. figure 3. The model requires the same upstream temperatures seen by the two sides of the SOL, i.e. inner and outer $T_{u, \text { in }}=T_{u, \text { out }}$, which directly results in $q_{\|, u, \text { in }}=q_{\|, u, \text { out }} \cdot L_{\|, \text {out }} / L_{\|, \text {in }}$. The upstream profiles for the inner and outer SOL differ only by a scalar factor and have the same decay length. Integration over the SOL width, with no assumption on the shape of the profiles, yields:

$P_{\text {in }} / P_{\text {out }}=L_{\|, \text {out }} / L_{\|, \text {in }}$

In addition to the assumed low target temperature, the model also neglects the effect of poloidal shifts of the stagnation point, which best approximates the upstream location, on the connection length.

The shape of the heat flux profile is derived for the outer SOL, but can be repeated in a similar fashion also for the inner SOL. We start by differentiating equation 11 with respect to $\rho$, with $L_{\|}$independent of $\rho$ :

$\frac{\partial T_{u}}{\partial \rho_{u}}=\frac{1}{\kappa_{0}}\left(\frac{7 q_{\|, u, \text { out }} L_{\|, \text {out }}}{2 \kappa_{0}}\right)^{-5 / 7} L_{\|, \text {out }} \frac{\partial q_{\|, u, \text { out }}}{\partial \rho_{u}}$

The radial derivative of the temperature is rewritten in terms of $P_{\perp}$ using equation 7 . After a further differentiation and substitutions using equations 8, 9 and 10 , we obtain a second order partial differential equation for the upstream parallel heat flux to one target (here the outer):

$$
\frac{\partial^{2} q_{\|, u, \text { out }}}{\partial \rho_{u}^{2}}=c_{1} q_{\|, \text {u, out }}^{12 / 7}+\frac{5}{7} q_{\|, u, \text { out }}^{-1}\left(\frac{\partial q_{\|, u, \text { out }}}{\partial \rho_{u}}\right)^{2}
$$

with

$$
\begin{aligned}
c_{1}= & \frac{B_{p, u}}{B_{\text {tot }, u}}\left(1+\frac{L_{\|, \text {out }}}{L_{\|, \text {in }}}\right) \frac{\kappa_{0}}{2 \pi a \sqrt{\frac{1+\kappa^{2}}{2}} \chi_{\perp} n_{e} L_{\|, \text {out }}} \\
& \times\left(\frac{7 L_{\|, \text {out }}}{2 \kappa_{0}}\right)^{5 / 7}
\end{aligned}
$$

The parallel connection length (set radially uniform across the entire SOL), $\chi_{\perp}$ and the total power crossing the separatrix $P_{\text {sep }}$ are among the free parameters.

The profile of the parallel heat flux upstream is assumed to be a decaying exponential:

$q_{\|, u, \text { out }}=q_{\|, u, \text { out }, \text { sep }} e^{-\rho / \lambda_{q}}$

with

$q_{\|, u, \text { out }, \text { sep }}=\frac{P_{\text {sep }} c_{d i v, \text { out }}}{2 \pi R_{u} \lambda_{q}} \frac{B_{\text {tot }, u}}{B_{p, u}}$

where $c_{\text {div, out }}$ is the fraction of power flowing to the outer target:

$c_{\text {div }, \text { out }}=\frac{P_{\text {out }}}{P_{\text {out }}+P_{\text {in }}}=\frac{L_{\|, \text {in }}}{L_{\|, \text {out }}+L_{\|, \text {in }}}$

The assumption of an exponential profile is supported by the shape of the numerical solution of equation 13 . Expression 15 is substituted in 13 and evaluated at the separatrix $(\rho=0)$. With $B_{p, u} \propto I_{P} / a$, one obtains the expression:

$$
\begin{aligned}
\lambda_{q} \propto & L_{\|, \text {out }}^{2 / 9}\left(1+\frac{L_{\|, \text {out }}}{L_{\|, \text {in }}}\right)^{-2 / 9} \chi_{\perp}^{7 / 9} n_{e, u}^{7 / 9} \\
& \times P_{\text {sep }}^{-5 / 9} I_{P}^{-2 / 9} B_{\text {tot }, u}^{2 / 9} a R_{u}^{5 / 9}
\end{aligned}
$$

The thermal diffusivity $\chi_{\perp}$, so far considered as a constant, may display a dependence on the upstream temperature. Two cases are explored:

- Bohm-like diffusivity for drift waves $\chi_{\perp} \propto \rho_{s} c_{s}$ [38], where $\rho_{s}$ is the ion Gyro radius and $c_{s}$ the ion sound speed. This yields $\chi_{\perp} \propto T_{u, s e p} / B_{t o t, u}$, therefore

$$
\begin{aligned}
\lambda_{q} \propto & L_{\|, \text {out }}^{4 / 11}\left(1+\frac{L_{\|, \text {out }}}{L_{\|, \text {in }}}\right)^{-4 / 11} n_{e, u}^{7 / 11} \\
& \times P_{\text {sep }}^{-3 / 11} I_{p}^{-4 / 11} B_{\text {tot }, u}^{-3 / 11} a R_{u}^{3 / 11}
\end{aligned}
$$

- Bohm-like diffusivity for ballooning modes $\chi_{\perp} \propto \rho_{s} c_{s} q$ [38], where $q=\left(a B_{\text {tot }, u}\right) /\left(R_{u} B_{\text {pol,u }}\right)$ is the safety factor. This yields $\chi_{\perp} \propto\left(T_{u, s e p} a^{2}\right) /\left(R_{u} I_{P}\right)$, therefore

$$
\begin{aligned}
\lambda_{q} \propto & L_{\|, \text {out }}^{4 / 11}\left(1+\frac{L_{\|, \text {out }}}{L_{\|, \text {in }}}\right)^{-4 / 11} n_{e, u}^{7 / 11} \\
& \times P_{\text {sep }}^{-3 / 11} I_{p}^{-1} B_{\text {tot }, u}^{4 / 11} a^{25 / 11} R_{u}^{-4 / 11}
\end{aligned}
$$




\subsection{Diffusive model for $S$}

The $S$ is defined by [31]:

$S=\int_{t}^{\mathrm{x}} \sqrt{\frac{\chi_{\perp}}{\chi_{\|}}} d l$

where the integration is performed along the path from X-point $(X)$ to target $(t)$, with $l$ defined as the parallel connection length starting at the target. The perpendicular diffusivity $\chi_{\perp}$ is assumed constant in the divertor. Using the Spitzer-Harm diffusivity $\chi_{\|}=\kappa_{0} T_{e}^{5 / 2} / n_{e}$, the $2 \mathrm{PM}$ relation for the temperature $T_{e}(l)=\left(\left(7 q_{\|} l\right) /\left(2 \kappa_{0}\right)\right)^{2 / 7}$ and requiring pressure conservation along each field line

$n_{e}(l) T_{e}(l)=n_{\mathrm{x}} T_{\mathrm{x}}$

one obtains:

$S=2 \sqrt{\frac{\chi_{\perp} n_{\mathrm{x}}}{\kappa_{0}}} T_{\mathrm{x}}^{-5 / 4} l_{\mathrm{x}}$

Further approximations are present in this derivation. Firstly, the equation for $T_{e}(l)$ is not strictly true over the entire SOL since $q_{\|}$varies along a field line following cross-field diffusion in the divertor. Secondly, equation 19 implies that the flow velocity in the SOL increases from zero to the ion sound speed at the target, in a infinitively thin layer.

\section{References}

[1] Loarte A et al. 2007 Nucl. Fusion 47203263 doi: 10.1088/0029-5515/47/6/S04

[2] Eich $\mathrm{T}$ et al. 2013 Nucl. Fusion $\mathbf{5 3} 093031$ doi: 10.1088/0029-5515/53/9/093031

[3] Coda S et al. 2017 Nucl. Fusion 57102011 doi: 10.1088/1741-4326/aa6412

[4] Verhaegh K et al. 2017 Nuclear Materials and Energy, in press doi: 10.1016/j.nme.2017.01.004

[5] Boedo J et al. 2009 Rev. Sci. Instrum 80123506 doi: $10.1063 / 1.3266065$

[6] Tsui C K et al. 2017 Phys. Plasmas 24062508 doi: $10.1063 / 1.4985075$

[7] Reimerdes H et al. 2017 Nucl. Fusion 57126007 doi: $10.1088 / 1741-4326 /$ aa82c2

[8] Harrison J et al. 2016 Nuclear Materials and Energy doi: 10.1016/j.nme.2016.10.020

[9] Theiler C et al. 2017 Nucl. Fusion 57072008 doi: 10.1088/1741-4326/aa5fb7

[10] Tsui C et al. 2016 58th APS Division of Plasma Physics Meeting doi:

[11] Herrmann A et al. 2001 28th EPS Conference on Controlled Fusion and Plasma Physics 2109-2112 doi:

[12] Pitts R et al. 1999 Nucl. Fusion 391433 doi: 10.1088/0029$5515 / 39 / 10 / 306$

[13] Eich $\mathrm{T}$ et al. 2011 Phys. Rev. Lett. 107215001 doi: 10.1103/PhysRevLett.107.215001

[14] Maurizio R et al. 2016 43rd EPS conference, Leuven, Belgium P4.027

[15] Theiler C 2017 SPC, Private communication (2017)

[16] Sheikh U et al. 2016 Review of Scientific Instruments 87 11D431 doi: 10.1063/1.4961271

[17] Makowski M et al. 2012 Phys. Plasmas 19056122 doi: $10.1063 / 1.4710517$
[18] Gallo A et al. 2016 Nuclear Materials and Energy doi: 10.1016/j.nme.2016.10.003

[19] Stangeby P C 2000 Plasma Phys. Control. Fusion 42 B271 doi: 10.1088/0741-3335/42/12B/321

[20] Chankin A et al. 1996 Plasma Phys. Control. Fusion 38 1579 doi: 10.1088/0741-3335/38/9/004

[21] Itami K et al. 1992 J. Nucl. Mater. 196755 - 758 doi: 10.1016/S0022-3115(06)80137-6

[22] Asakura N et al. 1995 J. Nucl. Mater. 220395 - 399 doi: 10.1016/0022-3115(94)00639-3

[23] Loarte A et al. 1999 J. Nucl. Mater. 266587 - 592 doi: 10.1016/S0022-3115(98)00590-X

[24] Leonard A et al. 1995 J. Nucl. Mater. 220325 - 329 doi: 10.1016/0022-3115(94)00493-5

[25] Hutchinson I et al. 1995 Plasma Phys. Control. Fusion 37 1389 doi: 10.1088/0741-3335/37/12/004

[26] Christen N et al. 2017 Plasma Phys. Control. Fusion 59 105004 doi: 10.1088/1361-6587/aa7c8e

[27] Gallo A et al. 2017 Accepted to Plasma Phys. Control. Fusion doi: 10.1088/1361-6587/aa857b

[28] Tamain P et al. 2016 Journal of Computational Physics 321 606-623 doi: 10.1016/j.jcp.2016.05.038

[29] Sieglin B et al. 2016 Plasma Phys. Control. Fusion 58 055015 doi: 10.1088/0741-3335/58/5/055015

[30] Spitzer L et al. 1953 Phys. Rev. 89 977-981 doi: 10.1103/PhysRev.89.977

[31] Nille D et al. 2016 arXiv:1610.04148v1 [physics.plasm-ph]

[32] Scarabosio A et al. 2015 J. Nucl. Mater. 463 49-54 doi: 10.1016/j.jnucmat.2014.11.076

[33] Faitsch M et al. 2017 Submitted to Plasma Phys. Control. Fusion doi:

[34] Scarabosio A et al. 2013 J. Nucl. Mater. 438 S426-S430 doi: 10.1016/j.jnucmat.2013.01.086

[35] Ahn J W et al. 2006 Plasma Phys. Control. Fusion 481077 doi: 10.1088/0741-3335/48/8/003

[36] Goldston R 2012 Nucl. Fusion 52013009 doi: 10.1088/0029-5515/52/1/013009

[37] Petrie T et al. 2013 J. Nucl. Mater. 438 S166-S169 doi: 10.1016/j.jnucmat.2013.01.051

[38] Ricci P et al. 2013 Phys. Plasmas 20010702 doi: $10.1063 / 1.4789551$ 